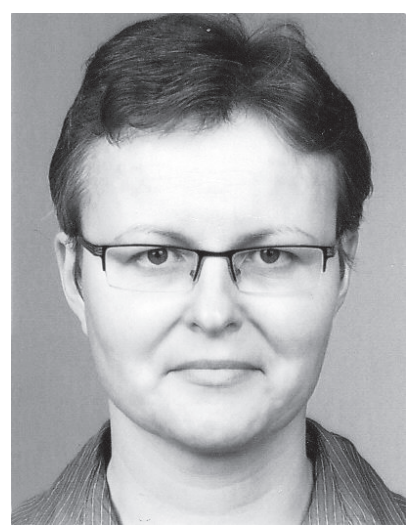

\section{Christiane Schiller}

Christiane Schiller - habilituota daktarè, Berlyno Humboldtų universiteto docentè.

Moksliniai interesai: Mažosios Lietuvos leksikografija, onomastika, kuršininkų kalba.

Adresas: Humboldt-Universität zu Berlin, Philosophische Fakultät II, Institut für Deutsche Sprache und Linguistik Historisch-Vergleichende Sprachwissenschaft 10099 Berlin. Tel.: 004930 2093-9725.

El. paštas: christiane.schiller@staff.hu-berlin.de.

Christiane Schiller: Privatdozentin at Berlin Humboldt University.

Research interests: Lexicography of Lithuania Minor, Onomastics, Kursenieku language.

Address: Humboldt-Universität zu Berlin, Philosophische Fakultät II, Institut für Deutsche Sprache und Linguistik Historisch-Vergleichende Sprachwissenschaft 10099 Berlin. Phone: 004930 2093-9725.

E-mail: christiane.schiller@staff.hu-berlin.de.

\title{
Humboldt-Universität zu Berlin
}

\section{KURONISMEN IM HALLESCHEN SOG. R I C H T ER - W ÖR T ER B U C H}

\author{
Anotacija \\ Straipsnyje tyrinejjami XVIII a. vokiečių-lietuvių kalbų žodyno (vad. Richterio žodynu) \\ kuronizmai, t. y. žodžiai, kurie priskiriami kuršininkų kalbai, vartotai Kuršių nerijoje ir \\ ị šiaurę nuo Klaipėdos iki 1945 m. Šie iki šiol mažai žinomi kuronizmai identifikuoja- \\ mi remiantis R. Pyčo „Deutsch-Kurisches Wörterbuch“, R. Pyčo, P. Kvaukos „Kurisches \\ Wörterbuch“, LKŽ ir K. Miūlenbacho ir J. Endzelyno „Lettisch-deutsches Wörterbuch“ \\ medžiaga.
}

PAGRINDINIAI ŽODŽIAI: kuronizmai, Mažosios Lietuvos žodynas, kalbų kontaktai.

\section{Abstract}

This article focuses on Curonisms, i.e. words originated from Latvian based dialects spoken on the Curonian Spit and to the north of Klaipeda until 1945, which were encountered in the so-called German-Lithuanian Richter-Wörterbuch from the $18^{\text {th }}$ century. These hitherto widely neglected Curonisms have been identified on the basis of the Deutsch-Kurisches Wörterbuch by Richard Pietsch, Kurisches Wörterbuch by Paul Kwauka and Richard Pietsch, the LKŽe and the Lettisch-deutsches Wörterbuch by Karl Mühlenbachs and Jānis Endzelīns.

KEYWORDS: curonisms, dictionary of Lithuania Minor, language contact. 
Im Archiv der Franckeschen Stiftungen zu Halle (Saale) wird unter der Signatur J 84 ein handschriftliches deutsch-litauisches Wörterbuch aufbewahrt, das in das Jahr 1728 datiert. Es ist benannt nach Johann Richter, dem ersten Dozenten für litauische Sprache am Litauischen Seminar in Halle, das dort von 1727 bis 1740 existierte. ${ }^{1}$ Erstmals beschrieben wurde dieses Wörterbuch 1935 von dem Hallenser Professor für vergleichende indogermanische Sprachwissenschaft, Franz Specht (Specht 1935). Auf ihn geht auch die Zuschreibung der Autorschaft an Johann Richter zurück. In den 1980er Jahren hat Vincas Urbutis das sog. Richter-Wörterbuch umfassender untersucht und ihm drei Aufsätze gewidmet (Urbutis 1987, Urbutis 1988a, Urbutis 1988b). In ihnen konnte Urbutis überzeugend die engen genetischen Beziehungen zu den anderen handschriftlich überlieferten deutsch-litauischen Wörterbüchern aufzeigen. Seitdem gilt Johann Richter nicht mehr als Autor des Wörterbuches, sondern lediglich als dessen Abschreiber. ${ }^{2}$

Das sog. Richter-Wörterbuch (weiterhin $R$ ) ist Bestandteil der preuBisch-litauischen lexikographischen Tradition. Enge genetische Beziehungen weist es, wie Urbutis $(1987,59 f f$.$) festgestellt hat, zum sog. Krau-$ se-Wörterbuch (weiterhin $K$ ), einer Handschrift aus der zweiten Hälfte des 18. Jahrhunderts, sowie zu dem nur fragmentarisch in Aufzeichnungen durch Kazimieras Būga überlieferten Wörterbuch Staatsarchiv Königsberg Msc. $844^{03}$ auf. Darüber hinaus gibt es genetische Beziehungen zu dem erst kürzlich von Ilja Lemeškin aufgefundenen sog. Prager Wörterbuch, einer Parallelhandschrift zu $K$ (Lemeškin 2011).

Von all den hier genannten Wörterbüchern unterscheidet sich $R$ durch eine Schicht von Žemaitismen, die bereits Franz Specht $(1935,48)^{4}$ bemerkte, und die Urbutis (1987) als Grund dafür dienten, $R$ als „klaipėdiškiu žodynas“ zu bezeichnen. Diese Dialektwörter stehen offenbar in Zusammenhang mit der Provenienz der Vorlage dieses Wörterbuches. Diese ist aus Memel über Königsberg nach Halle gelangt, wie der Briefwechsel zwi-

\footnotetext{
Ausführlich zum Litauischen Seminar in Halle s. Schiller 1994a, Schiller 1994b. Ausführlich zum sog. Richter-Wörterbuch s. Schiller 2010, 121-134.

3 Eine Rekonstruktion dieses seit dem Zweiten Weltkrieg verschollenen Wörterbuches bietet Zubaitienè 2009.

4 "Der Memelländische Einschlag läßt sich in dem Wörterbuch nicht verkennen [..] Žemaitischen Einfluß verraten ferner die mit linkay verstärkten Direktive [..]." (Specht 1935, 48).
} 
schen Georg Friedrich Rogall, Professor für Theologie an der Königsberger Universität und Gotthilf August Francke, Direktor der Franckeschen Stiftungen zu Halle und Sohn von August Herrmann Francke, einem der bedeutendsten Vertreter des Halleschen Pietismus, dokumentiert.

Aus einem Brief Rogalls vom Oktober 1727 an Gotthilf August Francke im Zusammenhang mit der Gründung des Litauischen Seminars in Halle geht hervor, dass Rogall ein Lexicon manuscriptum nach Halle geschickt hat:

[...] Sie werden ein lexicon manuscriptum nebst einigen Briefen an Herrn Richter und einige Katechismen finden, so mir Herr Erzpriester Pauli zugeschickt. [Wotschke $(1928,109)$ ]

Der in diesem Brief erwähnte Johann Arnold Pauli war seit 1713 Erzpriester in Memel (Krollmann 1967, 492). Die Herkunft der Vorlage aus Memel wird darüber hinaus auch durch Hinweise auf einen mehrfach im Text genannten Lehmann gestützt, wobei dieser Familienname vermutlich auf die Mitglieder der Memeler Pfarrerdynastie Lehmann zu beziehen ist. Aus dieser Dynastie sind im 16. und 17. Jahrhundert drei Pfarrer an der Litauischen Kirche in Memel hervorgegangen: Johann Lehmann der Ältere (1590-1664), Johann Lehmann der Jüngere (1630-1696) und Johann Theodor Lehmann (1655-1722) (Arnoldt 1777, 157).

Neben der mutmaßlichen Herkunft der Vorlage dieses Wörterbuches spielt für das Vorhandensein der Žemaitismen auch die Tatsache eine Rolle, dass auch der Abschreiber, Johann Richter, aus Memel stammte (Arnoldt 1777, 143).

In diesem Zusammenhang weitgehend unbeachtet geblieben ist eine Anzahl von eindeutigen Kuronismen, die im Mittelpunkt dieses Beitrages stehen sollen. Mit Kuronismen werden anders als in der sprachwissenschaftlichen Forschung üblich an dieser Stelle Lexeme bezeichnet, die dem Nehrungskurischen, also lettischbasierten Mundarten, die im nördlichen Ostpreußen verwendet wurden, zuzuordnen sind. Zwar hat bereits Franz Specht (1935, 48f.) festgestellt, dass es im Richter-Wörterbuch eine Phrase gibt, die gänzlich Lettisch sei, ${ }^{5}$ aber weder er noch Vincas Urbutis sind dieser Frage nachgegangen.

5 „Ganz lettisch mutet 270 laßt uns weiter, fort, vorwärts gehen ejsim projam an“ (Specht 1935, 48f.) 
Die Existenz von Kuronismen in einem Wörterbuch des Litauischen erscheint zunächst erklärungsbedürftig. Aber in Anbetracht dessen, dass eine Vorlage dieses Wörterbuches im Besitz eines an der Memeler Litauischen Kirche tätigen Geistlichen gewesen sein muss, verwundert diese Tatsache nicht. Nehrungskurisch wurde in Teilen des Pfarrbezirkes des Pfarrers gesprochen, in Memel selbst auf der Schmelz etwa und auf der Kurischen Nehrung, wo Memelsche litauische Diakone seit 1709 Seelsorge zu leisten hatten (Arnoldt 1777, 158).

In der Vergangenheit war die Kenntnis des Nehrungskurischen unter anderem ein Kriterium für die Besetzung von Pfarrstellen rund um das Kurische Haff. Sie ist überliefert etwa für den Bibelübersetzer, Johannes Bretke (1536-1602), Pfarrer in Labiau und Königsberg (Viktor Falkenhahn 1941, 50f.) und für Lazarus Sengstock (1562-1621), Pfarrer in Ruß, Memel und Königsberg und Herausgeber der Giesmes Chrikfczionifchkos ir Duchaunifchkos (1612) (Falkenhahn 1941, 448).

Kuronismen in litauischen Schriften sind bereits in älteren Sprachzeugnissen nachgewiesen worden. Sie sind mehrfach in den Schriften von Johannes Bretke untersucht worden, zuletzt von Jonas Palionis (Palionis 1991). Im Zusammenhang mit der preußisch-litauischen handschriftlichen Lexikographie sind sie bisher unbeachtet geblieben.

Ich bin auf diese Kuronismen im Zusammenhang mit der geplanten Edition des sog. Richter-Wörterbuches aufmerksam geworden. Für der Erstellung des Indexes der litauischen Äquivalente auf der Basis des Lietuviu kalbos žodynas (LKŽe) konnten die hier als Kuronismen klassifizierten Lexeme häufig dort nicht nachgewiesen werden; für andere war dort ein Hinweis auf das entsprechende lettische Äquivalent angeführt.

Dementsprechend sollen an dieser Stelle zwei Gruppen von Kuronismen unterschieden werden.

1. Kuronismen, die ausschließlich in entsprechenden Nachschlagewerken des Nehrungskurischen und des Lettischen verzeichnet sind und im LKŽe fehlen. Diese werden im Weiteren als primäre Kuronismen bezeichnet.

2. Kuronismen, die als lettische Lehnwörter im LKŽe verzeichnet sind. Diese werden im Weiteren als sekundäre Kuronismen bezeichnet. 
Allerdings wird bei vermeintlichen lettischen Lehnwörtern im LKŽe die Angabe der lettischen Form häufig nicht konsequent gehandhabt. So werden an dieser Stelle als sekundäre Kuronismen auch diejenigen Lexeme betrachtet, die Parallelen im Lettischen haben und anhand der Verbreitung in Grenznähe zum heutigen Lettland die Herkunft aus dem Lettisch wahrscheinlich erscheinen lassen. Für den Nachweis nehrungskurischer Herkunft haben die primären Kuronismen der Gruppe 1 die größte Aussagekraft.

Im Rahmen dieser Untersuchung wurden die in $R$ gefundenen $\mathrm{Ku}$ ronismen mit den entsprechenden lexikographischen Werken des Nehrungskurischen (Kwauka, Paul/ Pietsch, Richard: Kurisches Wörterbuch. [Lüneburg] 1987, weiterhin KW; Pietsch, Richard: Deutsch-Kurisches Wörterbuch. Lüneburg 1991, weiterhin DKW), sowie Lettischen (Mühlenbach, Karl: Lettisch-deutsches Wörterbuch; Redigiert, ergänzt und fortgesetzt von J. Endzelin. Riga 1923-1932, weiterhin $M E$ ) überprüft.

\section{Primäre Kuronismen}

Begonnen sei mit dem Beleg, der bereits Franz Specht (1935, 48f.) „ganz lettisch anmutete“.

(1) Eifim projam, laßt uns weiter, || fort, vorwärs gehen. $\left(R 270_{24}\right)$

Dieses Syntagma ist nicht ausschließlich nehrungskurisch, sondern hybrid, und enthält neben der litauischen Verbalform Eifim (1.pl.ft. zu eĩti 'gehen') das sowohl im Nehrungskurischen als auch im Lettischen verbreitete Adverb projam (vgl. pruoajum 'fort, weg' KWB 65, pruoajumiet 'fortgehen' KWB 65, pruoajume 'fort' DKW 125, pruoajum' 'weg' DKW 327; pruôjâm 'fort weg' $\mathrm{ME}_{3} 400$, pruôjām 'fort weg' (Preili) $\mathrm{ME}_{3} 400$ ).

(2) Lacken-Paallagge. Draballe. ( $R 1_{40}$ )

Das in Beleg 2 angeführte Paallagge ist durch seine Vokalgemination $<$ aa $>$ auffällig, die möglicherweise Intialakzent signalisieren soll. Diese Form stimmt überein mit dem in DKW 194 angeführten palage f. 'Laken', während KWB 57 die endungslose feminine Form palag f. 'Laken' bietet, wohingegen in ME die maskuline Form palags erscheint (vgl. palags 'Laken' ME3 54, palags 'Tischtuch' (Preili) $\mathrm{ME}_{3}$ 54).

(3) Knecht-Bernas. Puifzis. Algádzis. Szluzelninks. (R 140 40 
Der Beleg 3 enthält mit Puifzis und Algádzis gleich zwei vermeintliche Kuronismen, wobei nur Algádzis nach unserer Definition den primären Kuronismen zuzuordnen ist. Dieses Lexem ist in $\mathrm{ME}_{1} 54$ belegt (vgl. àlgãdzis 'Tagelöhner'), lässt sich allerdings nicht in den hier zugrunde gelegten Quellen für das Nehrungskurische, $K W B$ und $D K W$, nachweisen. $D K W$ führt das etymologisch verwandte algenieks m. 'Tagelöhner' an.

(4) Weile - Walanda [.] Adyna. Bride (eine kleine Weile) (R 276 ${ }_{16}$ )

Das in Beleg 4 angeführte Bride findet seinen Nachweis im DKW 329 vgl. brīde $f$. 'das Weilchen'. Das LKŽe verzeichnet hingegen nur die maskuline Form brỹdis mit Verweis auf das Lettische (vgl. brỹdis (la. brīdis) sm. (2) 'kartas, sykis, valandèlë), die ebenfalls $\mathrm{ME}$ bietet ( $\mathrm{ME}_{1} 333$ brìdis 'die Weile, eine kurze Zeit').

(5) Mancher - Tulimas, Tulas, Daźnas. Mem. dézis (l. deźis). ( $\left.R \quad 156_{27}\right)$

(6) Einer - Nekurfai. Zednas. Dazas. ( $R$ 68 ${ }_{36}$ )

(7) [Offt]mahl - Tankië̈. Dafznaÿ. Dazikarta. $\left(R \quad 174_{41}\right)$

Die Belege 5, 6 und 7 weisen auf lett. dažs und seine Ableitungen. In Beleg 5 wird das entsprechende Lexem dézis durch Mem. explizit dem Memeler Dialekt zugeordnet. Das in Beleg 6 angeführte Dazas (sofern nicht für Daznas verschrieben, wofür es jedoch keinen Anhaltspunkt gibt) zeigt eine an das Litauische angepasste Form der Endung. Das zugrunde zu legende Etymon dažs ist allerdings nur in ME belegt, vgl. $\mathrm{ME}_{1} 446$ dažs 'mancher'.

\section{Sekundäre Kuronismen}

Das in Beleg 7 angeführte Dazikarta ist im LKŽe mit Verweis auf das Lettische angeführt, vgl. dàžkart (la. dažkārt) adv. 'dažnaì. Allerdings scheint es in Litauen spärlich bezeugt. Außer in den Werken von Jurgis Pabrèža ist es nur im Lietuwiszkai-latwiszkai-lenkiszkai-rusiszkas žodynas. Tilžèje 1894 von Mykolas Miežinis nachgewiesen. In $K W B$ und $D K W$ ist dieses Lexem nicht verzeichnet, $\mathrm{ME}_{1} 446$ führt dažkàrt 'manchmal, zuweilen, oft' an.

(8) Knecht-Bernas. Puifzis. Algádzis. Szluzelninks. (R 140 40

Das in Beleg 8 angeführte Lexem Puifzis stimmt zur im LKŽe angeführten Form puĩ̌sis (la. puisis) sm. (2) 'vaikèzas' (vgl. $\mathrm{ME}_{3} 403$ puĩsis 'Knabe, Junge; unverheirateter Knecht') und weicht von den in $K W B$ und 
$D K W$ angeführten Formen ab, vgl. KWB 65 puišs m. 'Diener, Knecht', DKW 186 puišs m. 'Knecht, der' bzw. DKW 85, puišs m. 'Diener, der'.

(9) [Schwein]Stall - Kiaul[t]wartis. Kutis. $\left(\right.$ R 212 $\left.2_{41}\right)$

Die in Beleg 9 angeführte Form Kutis ist im LKŽ ohne Verweis auf das Lettische verzeichnet (vgl. kūtìs sf. (2) K. Būg, NdŽ, kūtis sm. N, [K]), doch weisen die aus den Mundarten angeführten Belege für diese Form auf die Gegend um Klaipeda. Die nehrungskurischen Wörterbücher bieten jeweils nur als maskulin ausgewiesene Formen (vgl. KWB kūt m. "kleiner Stall', DKW 278 kuts m. 'der Stall, kleiner Stall'; vgl. ME 338 kũts 'der Stall').

Žemaitische Züge wurden von dieser Wörterbuchredaktion bisher exklusiv $R$ zugeschrieben. Die vermeintlichen Kuronismen allerdings zeigen sich bereits teilweise in der dem sog. Richter-Wörterbuch und dem sog. Krause-Wörterbuch gemeinsamen Grundschicht. vgl. den Wörterbuchartikel $R \quad 128_{19}$ Hof - Dwaras. Padwarÿa. Muize, der mit K 2751 Hoff-Dwaras. Patwarija. Muyze. (abgesehen von orthographischen Abweichungen) identisch ist und somit auf eine gemeinsame Quelle zurückgeführt werden kann. Das in beiden Belegen angeführte Muize verzeichnet auch das LKŽe (vgl. muĩże (la. muiža) sf. (2) 'dvaras'), $\mathrm{ME}_{2}$ 682 muĩža 'das Landgut der Adligen, schöner Bauernhof. Es ist in KWD und DKW nicht verzeichnet.

Aber auch das sog. Krause-Wörterbuch und das sog. Prager-Wörterbuch zeigen in der von $R$ abweichenden gemeinsamen Ergänzungsschicht eindeutige Lemmata, die in ihrer Semantik auf die Gegend am Kurischen Haff weisen, so z.B. Termini aus dem Bereich der Fischereitechnologie.

K 341 Meerbufen - Ofts vel Ostas.

K 515 Sund-Oftas.

K 248 Haven - Oftas.

K 248 Im Hav[en] lauffen - J Ofta ibegti

K 341 Meeränge-Sufmauka. Saurumos.

K 479 Seegel auffftem [m]en - Ifprengti arduma.

K 479 Seegel auffziehen - Patraukti.

K 185 Fijcherkeul - Spurts.

K 295 Keule So die Fifcher gebrauch[en] die Fißche ins (korr. aus aus dem)\| Netz zu treiben. Spurts (korr. aus Sparts). 
Auf diese Gegend deuten aber auch vereinzelte Kuronismen in K. Dem in den genetisch verwandten Wörterbüchern noch ohne litauisches Äquivalent angeführten deutschen Lemma Seehund ist in $K$ das aus dem Lettischen stammende Lehnwort ronis zugeordnet, vgl. K 478 Seehund - Ronis. LKŽe verzeichnet rúonis (vgl. rúonis sm. (1) 'stambus irklakojis jūros žinduolis (Phoca)') außer in Lexika vor allem in den nordžemaitischen Dialekten. Die nehrungskurischen Wörterbücher bieten jeweils die Form ruoans m. (vgl. KWB 68 ruoans m. 'Seehund', DKW 268 ruoans m. 'der Seehund'. ME führt die Form ruônis an (vgl. ME 381 ruônis 'der Seehund').

Zum Abschluss soll auf den folgenden Beleg eingegangen werden.

K 341 Mausfahl-Palwas. Pelleks.

Zwar ist das litauische pelékas in vielen Dialekten in nicht unmittelbarer Grenznähe zu Lettland nachzuweisen (vgl. LKŽe: pelékas, -a adj. (1) J. Jabl, pelékas, -à (3) Jrb; 1. Q341, R, I, Rt, Grz, Vl, Skp, Ssk, Slm, Dgl pilkas kaip pelè (apie arkli, karvę): Pelèkà karvè K. Pilku plauku gyvuolys vadinas pelékas J. Ans nusipirko pelékq arklį Užv. Tokia graži pelèka telyčyté atsivesta Ds.), jedoch deutet die reduzierte Endung in das žemaitische Sprachgebiet und passt auch zu den für das Nehrungskurische überlieferten Formen (vgl. KWB 61 pêlêk 'grau', DKW 268 pêlêks 'grau'). Vgl auch $\mathrm{ME}_{3} 196$ pelêks 'grau'.

\section{Zusammenfassung}

Zusammenfassend kann festgestellt werden, dass einzelne Kuronismen Eingang in die preußisch-litauische Lexikographie gefunden haben. Ihre Existenz in diesen Wörterbüchern kann als Zeugnis des Sprachkontaktes zwischen dem Litauischen und dem Nehrungskurischen betrachtet werden. Durch die Möglichkeit diesen Sprachkontakt regional, d.h. am Kurischen Haff und um Memel (Klaipeda) zu lokalisieren, sind diese Kuronismen ein wichtiges Indiz für die Verortung der jeweiligen Wörterbücher und ihre mögliche Zuschreibung zu einem mutmaßlichen Autor bzw. Abschreiber. 


\section{Literatur}

Arnoldt 1777 - Daniel Heinrich Arnoldt. Kurzgefaßte Nachrichten von allen seit der Reformation an den lutherischen Kirchen Ostpreußens gestandenen Predigern. Königsberg: Hartung.

DKW - Richard Pietsch. Deutsch-Kurisches Wörterbuch. Lüneburg: Nordostdeutsches Kulturwerk, 1991.

K - Vokiečių-lietuvių kalbu žodynas. Lietuvių literatūros ir tautosakos institutas. Vilnius, Sign. F1-5792.

Krollmann 1967 - Christian Krollmann. Johann Arnold Pauli. Altpreußische Biographie, Bd. 2. Marburg: Elwert, 492.

KW - Paul Kwauka / Richard Pietsch. Kurisches Wörterbuch. [Lüneburg]: Nordostdeutsches Kulturwerk, 1987.

Lemeškin 2012 - Ilja Lemeškin. Friedrichui Wilhelmui Haackui priskiriamas Prahos rankraštinis vokiečių-lietuvių kalbų žodynas. Mažioji Lietuva: paribio kultūros tyrimai. Hrsg. von Grasilda Blažienè et al. Kalbų ir kultūru archyvai 3. Vilnius: Petro ofsetas, 31-58.

LKŽe - Lietuvių kalbos žodynas (I-XX, 1941-2002) 2005: elektroninis variantas / redaktorių kolegija: G. Naktinienè (vyr. redaktorè), J. Paulauskas, R. Petrokienė, V. Vitkauskas, J. Zabarskaitè. Vilnius: Lietuvių kalbos institutas. - www.lkz.lt.

ME - Karl Mühlenbach. Lettisch-deutsches Wörterbuch. Redigiert, ergänzt und fortgesetzt von J. Endzelin. Riga: Kultūras fonds, 1923-1932.

Palionis 1991 - Jonas Palionis. Lettizismen in Bretkūnas Wortschatz. Symposium Balticum. A Festschrift to honour Professor Velta Rūķe-Draviņa. Hrsg. von Baiba Metuzāle-Kangere / Helge Rinholm. Hamburg: Buske, 355-359.

$R$-Deutsch-Litauisches Lexikon. Franckesche Stiftungen, Halle a. d. Saale, Sign. AFrSt/H J 84.

Schiller 1994a - Christiane Schiller. Das Litauische Seminar in Halle (1727-1740) und seine Mitglieder. Auf Spurensuche. Acta Baltica XXXII (1994), 195-223.

Schiller 1994b - Christiane Schiller. Die Litauische Seminare in Königsberg und Halle. Eine Bilanz. Nordost-Archiv, N. F. Bd. III (1994) 2, 375-392.

Schiller 2010 - Christiane Schiller. Zur Geschichte der preußisch-litauischen Lexikographie. Die handschriftliche Lexikographie bis 1744. Habilitationsschrift. Humboldt-Universität zu Berlin 2010.

Specht 1935 - Franz Specht. Das Litauische Seminar an der Universität Halle. Archivum Philologicum 5 (1935), 36-53.

Urbutis 1987 - Vincas Urbutis. 1728 m. klaipèdiškių žodyno leksikografiniai šaltiniai. Baltistica 23 (1987) 1, 57-75.

Urbutis 1988a - Vincas Urbutis. 1728 m. klaipėdiškių žodyno orginalieji bruožai. Baltistica 24 (1988) 1, 80-93.

Urbutis 1988b - Vincas Urbutis. Dar apie 1728 m. klaipėdiškių žodyną. Baltistica 24 (1988) 2, 204-214.

Wotschke 1928 - Theodor Wotschke. Georg Friedrich Rogalls Lebensarbeit nach seinen Briefen. Königsberg: Beyer.

Zubaitienė 2009 - Vilma Zubaitienè. Neišlikusių XVIII a. Mažosios Lietuvos rankraštinių žodynu fragmentai. Vilnius: Lietuvių kalbos institutas. 


\section{Christiane Schiller}

\section{VAD. RICHTERIO ŽODYNO KURONIZMAI}

Santrauka

Halès Frankès įstaigose saugomame rankraštiniame vokiečių-lietuvių kalbų žodyne, kuris pagal savo nurašytoją vadinamas Richterio žodynu, buvo aptikta apie 10 kuronizmų, t. y. leksemų, kurios skirtinos Kuršių nerijos kuršininkų kalbai. Šių kuronizmų įtraukimas į žodyną gali būti aiškinamas žodyno originalo bei nurašytojo Johano Richterio kilme iš Klaipèdos.

Šie iki šiol mokslo beveik nepastebèti Prūsijos lietuvių rankraštinès leksikografijos, kuriai priklauso vad. Richterio žodynas, kuronizmai turi keleriopą reikšmę.

Pirmiausia jais dokumentuojami Kuršių nerijos kuršininkų kalbos faktai, kurių nėra gausiai paliudyta. Be to, jų egzistavimas rankraštiniuose lietuvių-vokiečių kalbų žodynuose - be vad. Richterio žodyno, kuronizmų rasta ir vad. Krauzès žodyne - gali būti lietuvių ir kuršininkų kalbų kontaktų liudininkas. Kadangi šiuos kontaktus galima regioniniu požiūriu lokalizuoti (t. y. Kuršiu marios ir Klaipeda), rastieji kuronizmai yra svarbus požymis, leidžiantis apibrèžti minètųjų žodynų sudarymo vietą ir priskirti spejjamam autoriui bei nurašytojui. 